九州大学学術情報リポジトリ

Kyushu University Institutional Repository

\title{
Inversion in geology by interactive evolutionary computation
}

WIJNS, CHRIS

CSIRO Exploration and Mining

MORESI, LOUIS

CSIRO Exploration and Mining

BOSCHETTI, FABIO

CSIRO Exploration and Mining

高木，英行

Kyushu Institute of Design

http://hdl. hand le. net/2324/4486299

出版情報 : 2001 IEEE International Conference on Systems, Man, and Cybernetics. 2, pp. 10531057, 2001-10-07. IEEE

バージョン:

権利関係 : $\odot 2001$ IEEE. Personal use of this material is permitted. Permission from IEEE must be obtained for all other uses, in any current or future media, including

reprinting/republishing this material for advertising or promotional purposes, creating new collective works, for resale or redistribution to servers or lists, or reuse of any copyrighted component of this work in other works. 


\title{
INVERSION IN GEOLOGY BY INTERACTIVE EVOLUTIONARY COMPUTATION
}

\author{
CHRIS WIJNS ${ }^{1, *}$, LOUIS MORESI ${ }^{1}$, FABIO BOSCHETTI $^{1}$, and HIDEYUKI TAKAGI ${ }^{2}$ \\ ${ }^{1}$ CSIRO Exploration and Mining, PO Box 437, Nedlands, WA 6009, Australia \\ ${ }^{2}$ Kyushu Institute of Design, Shiobaru, Minami-ku, Fukuoka 815-8540, Japan \\ E-Mail: chris,louis,fabio@ned.dem.csiro.au, takagi@kyushu-id.ac.jp \\ * now at Dept. of Geology and Geophysics, University of Western Australia, Nedlands, WA 6907, Australia
}

\begin{abstract}
We present the first step in the development of a system that would allow geological models to evolve backwards in time. The method of interactive evolutionary computation provides for the inclusion of geological knowledge and expertise in a rigorous mathematical inversion scheme, by simply asking an expert user to visually evaluate different geological models. We demonstrate the potential of the technique for the cases of folding and faulting.
\end{abstract}

\section{Keywords}

interactive evolutionary computation, inversion, geology.

\section{Introduction}

In recent years, fast computers have led to the development of quite sophisticated forward modelling of geological processes. We can answer questions such as "What faults or fractures will be generated by this stress field in this material ?", using accurate modelling of material behaviour. However, we really would like to solve the inverse problem, which is based upon field observations, i.e. "What stress field or material behaviour can generate these faults ?". Our task is thus to invert present-day observations in order to unravel the time evolution of a geological formation.

The first approach used by a geologist is to construct a time-dependant conceptual model in order to explain geological evolution. This is a heuristic method of inversion which is based upon an expert's knowledge and experience, but it is highly visual and usually offers little hard data. In our quest to ground such conceptual models in the laws of physics, we need to find the correct combination of initial conditions and material parameters in order to reproduce and thus validate the geologist's visual model. We lack numerical targets for mathematical inversion techniques, and so we have chosen a method of visual image ranking as a means for exploring geological parameter space. This approach capitalises upon the inherent subjectivity in geology.

\section{IEC-based Geological Inversion}

At present, geological modelling is almost exclusively confined to the forward modelling stage. The quality of a solution is often judged according to its resemblance to patterns seen in the field, to the fact that it does not contradict basic geological principles, or simply to the modeller's a priori expectations. Fit to data can be used as a further criterion when available, but this is rarely possible in a formal mathematical way.

Since geological simulation is computationally expensive, it is beneficial to employ some optimisation method to accelerate the search for satisfactory solutions. However, it is often hard to show, in a numerical sense, how solutions obtained are geologically suitable. Subjective judgment based on geological knowledge and expertise is usually necessary. This has driven us toward the use of some level of user interac- 
tivity in the process.

Interactive Evolutionary Computation (IEC) is an optimisation method that uses EC to optimise the target system according to human subjective evaluation. This technique has been widely used in artistic, engineering, educational, edutainment, and other fields for the past 10 years $[7,8,9]$. The IEC method is an EC approach that uses the capability of human evaluation as a fitness function. Unlike neural networks or other many nonlinear optimisation methods, IEC optimises a target system without the gradient information of the search space. This point is essential in order for IEC to be used for system optimisation based on subjective human evaluation.

Our two institutes have discussed and cooperated since 1998 in the area of geoscience and computational intelligence, and we have applied IEC to some geological problems $[2,3,4,5]$. We believe that the IEC system represents an advance on traditional, time-consuming trial and error approaches by providing a formal role for relevant geological experience and knowledge in inversion. The traditional numerical measure of data mismatch is replaced by the expert's subjective evaluation based on his or her geological knowledge and experience. The process works by producing different possible solutions and presenting them to the user for judgment and ranking.

Our IEC system is shown in Figure 1. It works by linking a geological forward model to a genetic algorithm (GA). A more detailed description of the specific GA implementation used in this work is described in [1]. The forward modelling code used here is a particle-in-cell finite element code $[6,10]$.

\section{Experiments}

We have applied the method to two geological situations, faulting and folding of the Earth's crust. The ranking of forward model results in the following cases is based upon comparison with a single target image. Although the forward models evolve in time, in this introduction to our inversion method, only the final configurations are used for visual evaluation.

\subsection{Faulting}

The first example seeks to reproduce common structures which arise when the Earth's crust undergoes ex-

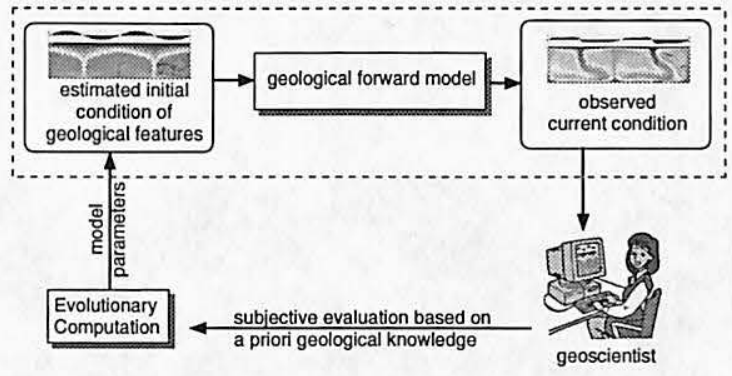

Figure 1: IEC-based geological forward modelling. The geological simulation evolves based upon an expert's knowledge and subjective judgments.

tension. The simple line sketch identified in Figure $2 a$ serves as our target image. The model is composed of two initially homogeneous crustal layers which are extended by applying a uniform velocity to the righthand boundary. This initial configuration is illustrated in Figure $2 b$.

The top of the box is filled with a low density, low viscosity background material which does not interfere with the mechanics of the problem. The upper layer of the crust has the property that increasing strain (deformation) causes material softening, and so strain tends to localise in areas of small perturbations. We are looking to form deformation bands which we interpret as faults due to extension. Although faults are, in reality, material discontinuities, in our continuum forward-modelling code we estimate that bands of high localised strain represent faults. Eight forward models are run at each step of the inversion, and six crustal strength parameters are allowed to vary.

Figure 2 illustrates the evolution of results using the IEC algorithm. Accumulated strain (faulting) is indicated by areas of darkened material. The first panel (i) contains no models which resemble the target image. In fact, only two out of the eight models have converged numerically and extended to full length, the others failing to do so because of unstable material parameter combinations. Models 6 and 8 exhibit structures penetrating the upper layer, and for this reason they are ranked first and second, respectively. The other models do not merit ranking, but are nonetheless weighted randomly by the GA in order to fill up the remaining six positions. 


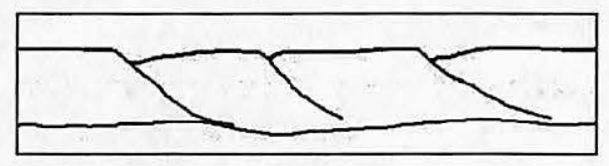

a

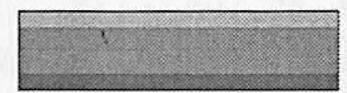

Rank:

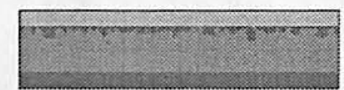

Rank:

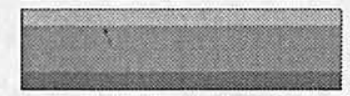

Rank:

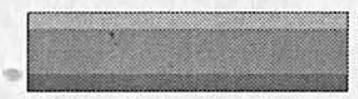

Rank:

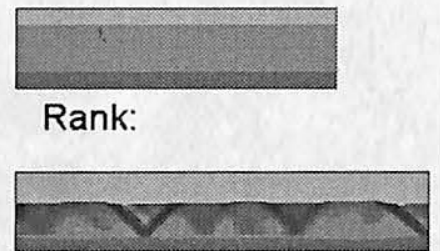

Rank: 1

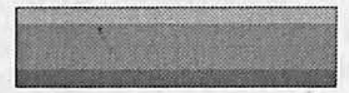

Rank:

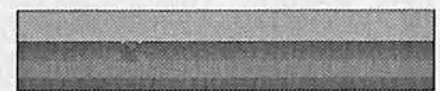

Rank: 2

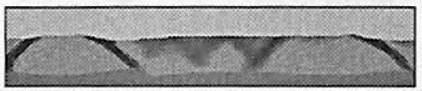

Rank: 1

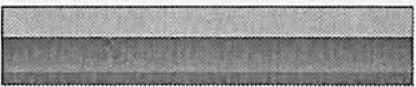

Rank: 5

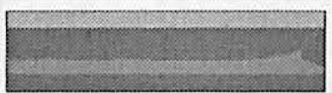

Rank:

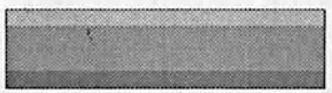

Rank:

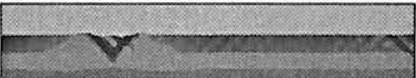

Rank: 3

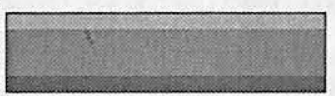

\section{Rank:}

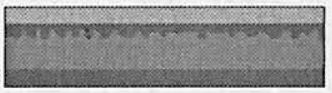

Rank:

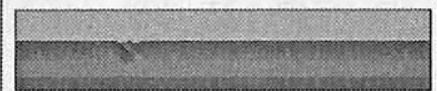

Rank: 4

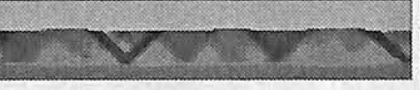

Previous best - Rank: 2

ii b

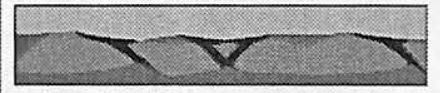

Rank: 2

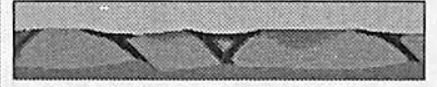

Rank: 5

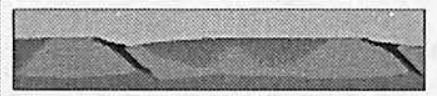

Rank: 1

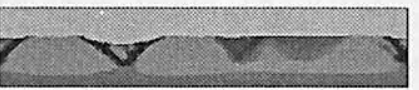

Rank: 6

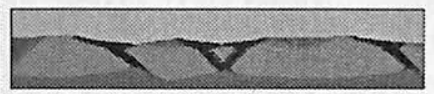

Rank: 4

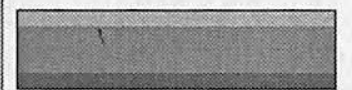

Rank: 9

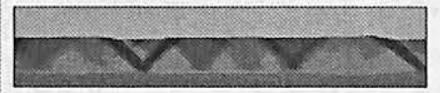

Rank: 7

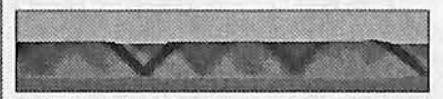

Rank: 8

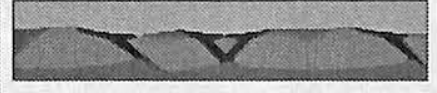

Previous best - Rank: 3

iii

Figure 2: Target image (a), initial geometry of the crust (b), and evolution of the IEC inversion for the faulting problem. Panels (i) to (iii) represent the first two and the last generation of the GA. Images are ranked according to their similarity with the target image. Some models have not been extended to full length because of numerical non-convergence. 


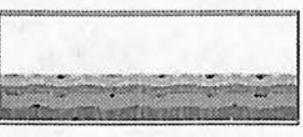

a

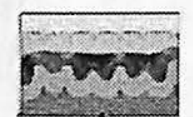

Rank: yes

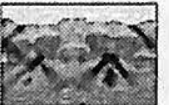

Rank: yes

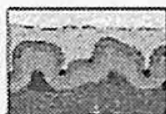

Rank: yes

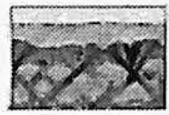

Rank: yes

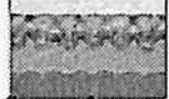

Rank: no

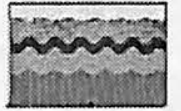

Rank: no

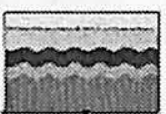

Rank: no

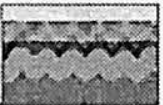

Rank: no

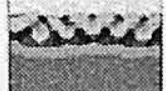

Rank: no

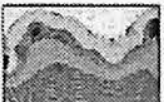

Rank: yes

b

Figure 3: Initial slightly perturbed layers for the folding problem (a), and final generation of the GA (b).

Ranking simply includes or excludes the result from the category of multiple wavelength folding.

Panel ii contains the second iteration of the algorithm. Once again there are four models which do not converge numerically, but those that do converge generally display more crustal-scale structures than in the first iteration. The rank of each model result is noted below each image. We continue iterating in this manner a total of six times, at which point half of the resulting images are qualitatively similar to the target image (panel iii), and the process is halted. The outcome is a set of crustal strength parameters that leads to the behaviour observed and inferred in the field.

\subsection{Folding}

In this next problem, we would like to promote the development of multiple simultaneous folding wavelengths as a box of layered material (Figure 3a) is compressed from one end. There is an initial wavelength present, and we look for the appearance of at least one more wavelength, in contrast to observing only the passive amplification of the initial perturbation. We do not refer to any target image, as in this example our target is deliberately more vague. The behaviour we seek will depend upon the particular material property contrasts. We allow the GA to vary the layer viscosities and thicknesses as well as their yield stresses.

Figure $3 \mathrm{~b}$ shows the fifth and final generation of the algorithm, in which half of the outcomes display multiple wavelengths of folding. An analysis of the input parameters shows us that such behaviour depends upon the presence of at least one layer with high viscosity and yield stress (i.e. strong) and at least one layer which is substantially weaker. The variety of different results which is deemed acceptable is indicative of the variation in the other parameters, i.e. which layers are strong or weak, and relative thicknesses.

\section{Discussion}

For either of the above problems, arriving at a suitable combination of parameters would previously have involved one of two more laborious approaches: the manual selection of parameters by trial and error, or an exhaustive coverage of all parametric space. Trial and error may succeed with a limited number of param- 
eters, but depends upon the user's knowledge of the coupling and feedback between parameters, which, in highly non-linear problems involving complex crustal rheologies, may be impossible. A parametric study quickly becomes unfeasible due to the sheer number of models which must be run as the number of parameters is increased. Neither of these approaches takes full advantage of the expert knowledge of an experienced geologist.

Our IEC method can be used by a field expert to invert for model parameters through the comparison of suitable images. The first case, involving faulting of the crust, is an example of a specific inversion target where an image has replaced a numerical target. The folding example illustrates the use of this algorithm as a classification tool, where a ranking is arguable. The GA graduallly accumulates more models in the appropriate (subjective) category. The analysis of the results in this case is more complicated and involves sifting through the history of the different generations.

Since all forward models evolve in time, the ranking procedure may be made more sophisticated by considering the evolution of the structures involved. Multiple processes may lead to the same outcome, thus the consideration of evolutionary history reduces the nonuniqueness of the inversion solution.

\section{Conclusions}

The technique of IEC has considerably diminished the effort required to explore parameter space during the inversion of conceptual models in geology. We bypass the lack of numerical data for an inversion target by using a GA together with image ranking to focus on a visual target. This approach exploits the experience and knowledge of an expert user in a visual and therefore intuitive environment.

\section{References}

[1] F. Boschetti, M. Dentith, R. and List, Inversion of seismic refraction data using genetic algorithms, Geophysics, pp. 1715-1727, 1996.

[2] F. Boschetti, L. Moresi, and K. Covil, Interactive inversion in geological applications, Intl. Conf. on Knowledge-based Intelligent Information En- gineering Systems (KES'99), Adelaide, Australia, pp.276-279, Aug./Sept. 1999.

[3] F. Boschetti and L. Moresi, Comparison between interactive (subjective) and traditional (numerical) inversion by genetic algorithms, Congress on Evolutionary Computation (CEC2000), La Jolla, CA, USA, pp.522-528, July 2000.

[4] F. Boschetti and H. Takagi, Visualization of EC landscape to accelerate EC conversion and evaluation of its effect, Congress on Evolutionary Computation (CEC2001), Seoul, Korea, pp.880-886, May 2001.

[5] F. Boschetti and L. Moresi, Interactive inversion in geosciences, Geophysics, in press 2001.

[6] L.-N. Moresi and V.S. Solomatov, Numerical investigation of $2 \mathrm{D}$ convection with extremely large viscosity variations, Physics of Fluids, vol. 7, no. 9, pp.155-164, 1995.

[7] H. Takagi, Interactive evolutionary computation: system optimization based on human subjective evaluation, IEEE Intl. Conf. on Intelligent Engineering Systems (INES'98), Vienna, Austria, pp.1-6, Sept. 1998.

[8] H. Takagi, Interactive evolutionary computation cooperation of computational intelligence and human KANSEI -, 5th Intl. Conf. on Soft Computing (IIZUKA'98), lizuka, Fukuoka, Japan, World Scientific: Singapore, pp.41-50, Oct. 1998.

[9] H. Takagi, Interactive evolutionary computation: fusion of the capacities of EC optimization and human evaluation, Proceedings of the IEEE, in press 2001.

[10] http://www.ned.dem.csiro.au/research/solidMech /PIC/Ellipsis.htm. 